\title{
A Galerkin Approach for Modelling the Pantograph-Catenary Interaction
}

\author{
John Martin and Stephen Duncan \\ Department of Engineering Science \\ University of Oxford \\ john.martin@eng.ox.ac.uk \\ stephen.duncandeng.ox.ac.uk
}

\begin{abstract}
The pantograph-catenary interaction was modelled for high-speed electric and hybrid trains. A lumped-mass pantograph model was used and the overhead wires were modelled as Euler-Bernoulli beams. Each vertical and horizontal wire deflection was decomposed into an infinite series of spatial basis functions, which were chosen to be the eigenmodes of the Euler-Bernoulli PDE, and corresponding time functions. The boundary conditions were used to evaluate the spatial basis functions and reduce the PDEs to ODEs in terms of the time functions. Elimination of variables was used to remove the algebraic contact constraints and reduce the overall index-three DAE to an ODE. This linear, time-varying ODE was solved by integration and the elimination process was reversed in order to recover the original states. The Simulink model was validated against the 2002 and 2018 European Standards, BS:EN 50318:2002 and BS:EN 50318:2018 respectively. In both cases, the model produced accurate results with exceptional simulation speeds.
\end{abstract}

\section{Introduction}

Electric and hybrid trains are popular across Europe and many further electrification projects are under way. As train speeds increase, it is important to design pantographs and catenary systems that robustly provide a suitable contact force at higher operating speeds. If the contact force is too large, this will cause excessive wear on both the contact wire and the pantograph head; if the contact force is too small, there is a risk of loss of contact, which interrupts power delivery and causes arcing. Since many configurations must be tested and real line tests are expensive, accurate modelling of the pantograph-catenary interaction is a necessary part of overhead-line-equipment (OLE) design.

In [3], the results from ten simulation programs were compared and used to create the standard BS:EN 50318:2018. The majority of previous pantograph-catenary simulation software has used finite-element methods (FEM) $[1,7,8]$. Two prominent programs are PCaDA and OSCAR, which both use FEM to model the overhead system, lumped-mass models for the pantograph, the Euler-Bernoulli beam equation for the overhead wires and a penalty system to keep the height of the pantograph head and the contact-wire deflection in agreement. This paper takes an alternative approach by using a Galerkin method instead of FEM and an algebraic contact constraint instead of a penalty method. Similar algebraic constraints are also used to maintain contact between the registration arms and the contact wire.

Simulations based on FEM provide excellent accuracy close to the point of contact, but the speed of simulation is poor. It is desirable to reduce simulation time so that multiple OLE set-ups and 'what if?' scenarios can be tested. This paper presents pantograph-catenary modelling code, running in MATLAB and Simulink, that aims to greatly improve the simulation speed while maintaining high accuracy.

\section{The Galerkin Approach}

A Galerkin approach, also known as modal analysis or spectral analysis, has been used to solve the catenarywire PDEs. This approach is explained in [4]: for each wire, the vertical and horizontal deflections are each separated into an infinite series of spatial basis functions and corresponding time functions, where the spatial basis functions are chosen to be the eigenfunctions of the PDE. The eigenfunctions are calculated using the boundary conditions and the PDEs are reduced to ODEs, with the amplitudes of the modes as the states. After integration, the evaluated time functions are recombined with the spatial basis functions to give vertical and horizontal wire deflections as functions of space and time.

The main incentive to use a Galerkin approach over FEM is to improve the speed of simulation. The infinite series of eigenfunctions is truncated to match the frequency response of the pantograph. Since the reciprocal of the maximum spatial frequency is typically more than an order of magnitude larger than the 
distance between FEM elements for the same overhead system, the Galerkin approach typically requires fewer than one tenth of the number of states to be evaluated. This leads to improved simulation speed, allowing more overhead-system configurations to be modelled in a given timeframe.

Another advantage of this software is that it can take an arbitrary velocity or acceleration profile as an input. This means that realistic velocity profiles can be tested, rather than requiring constant velocity.

The approach is evaluated using standardised pantograph-catenary simulations. It is shown that this Galerkin approach yields accurate solutions with good simulation speed and can handle multiple pantographs, dropper unloading and the transition from one contact wire to the next.

\section{Mathematical Modelling}

The mathematical model included two pantographs in contact with a single contact wire. A simplified model was used for validation against the 2002 European Standard and the full model was used for the 2018 standard.

\subsection{The Pantographs}

A lumped-mass pantograph model was used, as shown in Figure 1. The mass-spring-damper constants were specified by the European Standards and were chosen in order to match the frequency characteristics of the real pantograph. A constant uplift force, $P_{1}$, is applied to the base of the pantograph by a pneumatic actuator in order to maintain contact between the pantograph and the contact wire.

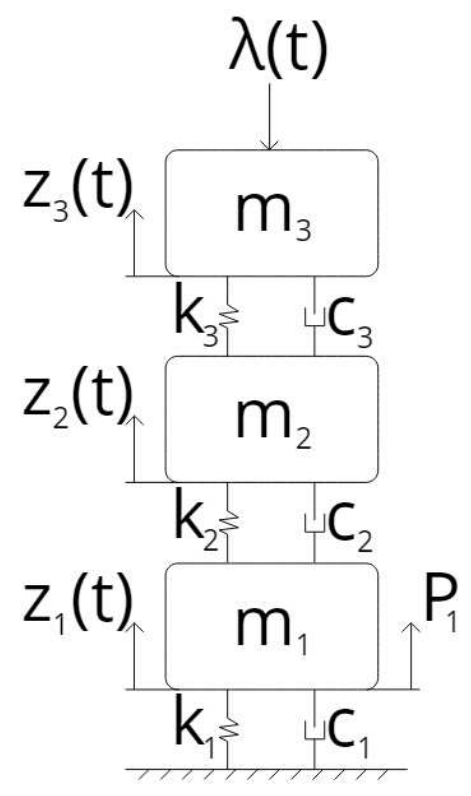

Fig. 1: Diagram of lumped-mass pantograph model

$$
\begin{gathered}
m_{1} \ddot{z}_{1}(t)=-\left(c_{1}+c_{2}\right) \dot{z}_{1}(t)+c_{2} \dot{z}_{2}(t)-\left(k_{1}+k_{2}\right) z_{1}(t)+k_{2} z_{2}(t)+P_{1} \\
m_{2} \ddot{z}_{2}(t)=c_{2} \dot{z}_{1}(t)-\left(c_{2}+c_{3}\right) \dot{z}_{2}(t)+c_{3} \dot{z}_{3}(t)+k_{2} z_{1}(t)-\left(k_{2}+k_{3}\right) z_{2}(t)+k_{3} z_{3}(t) \\
m_{3} \ddot{z}_{3}(t)=c_{3} \dot{z}_{2}(t)-c_{3} \dot{z}_{3}(t)+k_{3} z_{2}(t)-k_{3} z_{3}(t)-\lambda(t)
\end{gathered}
$$

where $z_{i}(t)$ is the height of the $i^{t h}$ pantograph block, $m_{i}$ the mass of the $i^{\text {th }}$ pantograph block, $c_{i}$ the $i^{t h}$ damper constant, $k_{i}$ the $i^{t h}$ spring constant, $P_{1}$ the constant uplift force and $\lambda(t)$ the contact force between the pantograph head and the contact wire. 


\subsection{The Overhead Wires}

The contact wire and messenger wire were modelled as Euler-Bernoulli beams. The length of each wire was $550-600 \mathrm{~m}$ and the radius was only $6.2-7.0 \mathrm{~mm}$, so the beams were extremely slender and bending moments were negligible.

\section{Contact Wire - Vertical}

$$
\rho A \frac{\partial^{2} w(x, t)}{\partial t^{2}}=-E I \frac{\partial^{4} w(x, t)}{\partial x^{4}}+P \frac{\partial^{2} w(x, t)}{\partial x^{2}}-\rho A g+\sum_{l=1}^{2}\left(\lambda_{l}(t) \delta\left(x-x_{\mathrm{p}, l}(t)\right)\right)
$$

where $x$ is the horizontal position, $t$ time, $x_{\mathrm{p}, l}(t)$ the horizontal position of the $l^{\text {th }}$ pantograph, $w(x, t)$ the vertical contact-wire deflection, $\rho$ the wire density, $A$ the cross-sectional area, $E$ Young's modulus, $I$ the second moment of area, $P$ the tension, $g$ the gravitational constant, $\lambda_{l}(t)$ the contact force between the $l^{t h}$ pantograph and the wire and $\delta(x)$ a Dirac Delta function.

The terms on the right-hand side, from left to right, are the bending stiffness, axial tension, self-weight and contact force. The pantograph exerts moving a point force on the contact wire, which is represented by a Dirac Delta function, because the Euler-Bernoulli beam equation is written in terms of force per unit length. The pantograph exerts no horizontal force and is never in contact with the messenger wire, so this contact-force term does not appear in the horizontal contact-wire equation or messenger-wire equation.

\section{Contact Wire - Horizontal}

$$
\rho A \frac{\partial^{2} w^{p}(x, t)}{\partial t^{2}}=-E I \frac{\partial^{4} w^{p}(x, t)}{\partial x^{4}}+P \frac{\partial^{2} w^{p}(x, t)}{\partial x^{2}}
$$

where $w^{p}(x, t)$ is the horizontal contact-wire deflection.

\section{Messenger Wire - Vertical}

$$
\rho^{\mathrm{m}} A^{\mathrm{m}} \frac{\partial^{2} w_{i}^{\mathrm{m}}(x, t)}{\partial t^{2}}=-E^{\mathrm{m}} I^{\mathrm{m}} \frac{\partial^{4} w_{i}^{\mathrm{m}}(x, t)}{\partial x^{4}}+P^{\mathrm{m}} \frac{\partial^{2} w_{i}^{\mathrm{m}}(x, t)}{\partial x^{2}}-\rho^{\mathrm{m}} A^{\mathrm{m}} g
$$

where $x$ is the horizontal position in the messenger span's coordinate system, $\rho^{\mathrm{m}}$ the messenger-wire density, $A^{\mathrm{m}}$ the cross-sectional area, $w_{i}^{\mathrm{m}}(x, t)$ the vertical deflection of the $i^{t h}$ messenger span, $E^{\mathrm{m}}$ Young's modulus, $I^{\mathrm{m}}$ the second moment of area and $P^{\mathrm{m}}$ the axial tension.

\section{Messenger Wire - Horizontal}

The messenger-wire's horizontal deflection was assumed to be static and piecewise linear, because no dynamic forces were exerted on the messenger wire. Each messenger-wire span stretched horizontally from one support to the next in a straight line.

\subsection{The Droppers}

The droppers were modelled as linear springs when in tension and were ignored when in compression. This was achieved by assuming that all droppers were in tension, then applying additional forces to remove the tensions of any unloaded droppers. The dropper tensions were given by (7) and were added to (4) and (6) as point forces, giving (8) and (9), respectively.

$$
F_{\mathrm{d}, i, j}(t)=k_{\mathrm{d}, i, j}\left(w_{i}^{\mathrm{m}}\left(x_{\mathrm{d}, i, j}^{\mathrm{m}}, t\right)-w\left(x_{\mathrm{d}, i, j}, t\right)\right)
$$

where $F_{\mathrm{d}, i, j}(t)$ is the tension in the $j^{t h}$ dropper of the $i^{t h}$ messenger span, $k_{\mathrm{d}, i, j}$ the spring constant of the dropper, $x_{\mathrm{d}, i, j}$ the horizontal position of the dropper in the contact wire's coordinate system and $x_{\mathrm{d}, i, j}^{\mathrm{m}}$ the horizontal position of the dropper in the $i^{\text {th }}$ messenger span's coordinate system.

$$
\begin{aligned}
\rho A \frac{\partial^{2} w(x, t)}{\partial t^{2}}= & -E I \frac{\partial^{4} w(x, t)}{\partial x^{4}}+P \frac{\partial^{2} w(x, t)}{\partial x^{2}}-\rho A g+\lambda(t) \delta\left(x-x_{\mathrm{p}}(t)\right) \\
& +\sum_{i=1}^{N^{\mathrm{m}}} \sum_{j=1}^{N_{\mathrm{d}}}\left(k_{\mathrm{d}, i, j}\left(w_{i}^{\mathrm{m}}\left(x_{\mathrm{d}, i, j}^{\mathrm{m}}, t\right)-w\left(x_{\mathrm{d}, i, j}, t\right)\right) \delta\left(x-x_{\mathrm{d}, i, j}\right)\right)
\end{aligned}
$$


where $N_{\mathrm{d}}$ is the number of droppers on the messenger span.

$$
\begin{aligned}
\rho^{\mathrm{m}} A^{\mathrm{m}} \frac{\partial^{2} w_{i}^{\mathrm{m}}(x, t)}{\partial t^{2}} & =-E^{\mathrm{m}} I^{\mathrm{m}} \frac{\partial^{4} w_{i}^{\mathrm{m}}(x, t)}{\partial x^{4}}+P^{\mathrm{m}} \frac{\partial^{2} w_{i}^{\mathrm{m}}(x, t)}{\partial x^{2}}-\rho^{\mathrm{m}} A^{\mathrm{m}} g \\
& +\sum_{j=1}^{N_{\mathrm{d}}}\left(k_{\mathrm{d}, i, j}\left(w\left(x_{\mathrm{d}, i, j}, t\right)-w_{i}^{\mathrm{m}}\left(x_{\mathrm{d}, i, j}^{\mathrm{m}}, t\right)\right) \delta\left(x-x_{\mathrm{d}, i, j}^{\mathrm{m}}\right)\right)
\end{aligned}
$$

\subsection{The Registration Arms}

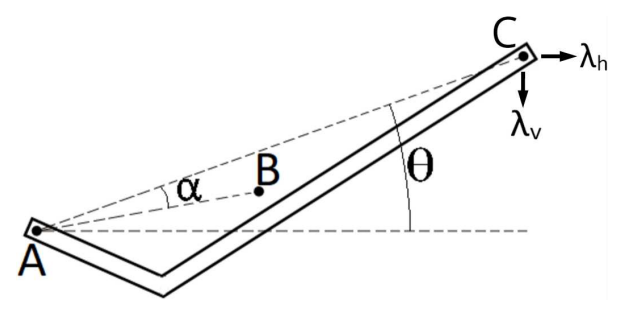

Fig. 2: Model of registration arm. A is the static support, B is the centre of mass and $\mathrm{C}$ is the point of contact with the contact wire.

$$
J_{\mathrm{r}, k} \ddot{\theta}_{k}(t)=-L_{\mathrm{r}, k} \lambda_{\mathrm{v}, k}(t) \cos \theta_{k}(t)-L_{\mathrm{r}, k} \lambda_{\mathrm{h}, k}(t) \sin \theta_{k}(t)-m_{\mathrm{r}, k} g \frac{L_{\mathrm{g}, k}}{2} \cos \left(\theta_{k}(t)-\alpha_{k}\right)
$$

where $J_{\mathrm{r}, k}$ is the moment of intertia of the $k^{t h}$ registration arm, $\theta_{k}(t)$ the pitch of the arm, $L_{\mathrm{r}, k}$ the length of the arm, $\lambda_{\mathrm{v}, k}(t)$ the upward force exerted on the contact wire by the arm, $\lambda_{\mathrm{h}, k}(t)$ the horizontal force exerted on the contact wire by the arm, $m_{\mathrm{r}, k}$ the mass of the arm, $L_{\mathrm{g}, k}$ the distance from the arm's support to its centre of mass and $\alpha_{k}$ the angle of the centre of mass.

$$
\begin{gathered}
\rho A \frac{\partial^{2} w(x, t)}{\partial t^{2}}=-E I \frac{\partial^{4} w(x, t)}{\partial x^{4}}+P \frac{\partial^{2} w(x, t)}{\partial x^{2}}-\rho A g+\sum_{k=1}^{N_{\mathrm{r}}}\left(\lambda_{\mathrm{v}, k}(t) \delta\left(x-x_{\mathrm{r}, k}\right)\right) \\
+\sum_{i=1}^{N^{\mathrm{m}}} \sum_{j=1}^{N_{\mathrm{d}}}\left(k_{\mathrm{d}, i, j}\left(w_{i}^{\mathrm{m}}\left(x_{\mathrm{d}, i, j}^{\mathrm{m}}, t\right)-w\left(x_{\mathrm{d}, i, j}, t\right)\right) \delta\left(x-x_{\mathrm{d}, i, j}\right)\right)+\lambda(t) \delta\left(x-x_{\mathrm{p}}(t)\right)
\end{gathered}
$$

where $x_{\mathrm{r}, k}$ is the horizontal position of the $k^{t h}$ registration arm in the contact wire's coordinate system.

$$
\begin{aligned}
\rho A \frac{\partial^{2} w^{p}(x, t)}{\partial t^{2}}= & -E I \frac{\partial^{4} w^{p}(x, t)}{\partial x^{4}}+P \frac{\partial^{2} w^{p}(x, t)}{\partial x^{2}} \\
& +\sum_{k=1}^{N_{\mathrm{r}}}\left(\operatorname{sign}\left(s_{k}\right) \lambda_{\mathrm{h}, k}(t) \delta\left(x-x_{\mathrm{r}, k}\right)\right)
\end{aligned}
$$

where $s_{k}$ is the horizontal stagger of the point of contact between the registration arm and the contact wire when the arm is horizontal.

\subsection{The Algebraic Constraints}

\section{The Horizontal Registration-Arm Constraints}

$$
w^{p}\left(x_{\mathrm{r}, k}, t\right)=s_{k}+L_{\mathrm{r}, k} \operatorname{sign}\left(s_{k}\right)\left(1-\cos \theta_{k}(t)\right)
$$

\section{The Vertical Registration-Arm Constraints}

$$
w\left(x_{\mathrm{r}, k}, t\right)=L_{\mathrm{r}, k} \sin \theta_{k}(t)
$$




\section{The Pantograph Position Constraints}

One algebraic constraint must be satisfied for each of the two pantographs. These constraints reflect the fact that the pantographs remain in contact with the contact wire, which means that the height of each pantograph head must match the contact-wire height at that horizontal position.

$$
z_{3, l}(t)+\bar{z}_{3, l}=w\left(x_{\mathrm{p}, l}(t), t\right) \quad l=1,2
$$

where $\bar{z}_{3, l}$ is the steady-state height of the $l^{t h}$ pantograph head, with no uplift force applied.

\subsection{Spatial Discretisation}

Each wire deflection was decomposed into an infinite series of spatial basis functions, $\psi_{n}(x)$, and corresponding time functions, $q_{n}(t)$.

$$
\begin{aligned}
w(x, t) & =\sum_{n=1}^{\infty}\left(q_{n}(t) \phi_{n}(x)\right) \\
w^{p}(x, t) & =\sum_{n=1}^{\infty}\left(p_{n}(t) \phi_{n}(x)\right) \\
w_{i}^{\mathrm{m}}(x, t) & =\sum_{n=1}^{\infty}\left(q_{i, n}^{\mathrm{m}}(t) \phi_{i, n}(x)\right) \quad i=1,2,3, \ldots N_{\mathrm{m}}
\end{aligned}
$$

The $\psi_{n}$-terms were evaluated using the boundary conditions for the contact wire and for each messengerwire span. Then, (11), (12) and (9) were rearranged in terms of $q_{n}(t), q_{n}^{\mathrm{m}}(t)$ and $p_{n}(t)$, respectively.

\subsection{The Index-Three DAE}

The equations of motion for the dynamics of the pantographs, overhead wires and registration arms were compiled into one ODE. This was combined with the algebraic constraints to yield (17). Since the pantograph position constraint could be differentiated three times in order to reduce this DAE to an ODE, the differential index is three.

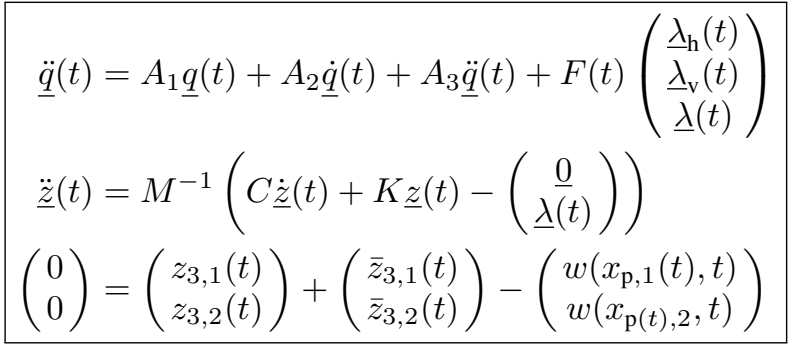

$$
\begin{aligned}
& \underline{q}(t)=\left(\begin{array}{lllllll}
\underline{\theta}^{\mathrm{T}}(t) & \underline{q}^{\mathrm{T}}(t) & \underline{p}^{\mathrm{T}}(t) & \underline{q}_{1}^{\mathrm{mT}}(t) & \underline{q}_{2}^{\mathrm{mT}}(t) & \cdots & \left.\underline{q}_{N_{\mathrm{m}}}^{\mathrm{mT}}(t)\right)^{\mathrm{T}}
\end{array}\right.
\end{aligned}
$$

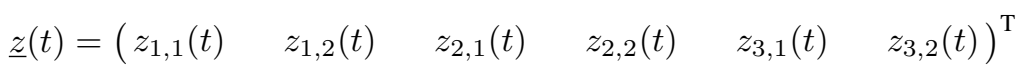

where the matrices $A_{1}, A_{2}, A_{3}, F(t), M, C$ and $K$ were evaluated using the previously derived ODEs.

\subsection{Elimination of Variables}

In order to solve the DAE, the constraint equations were removed by elimination of variables. Each constraint, as well as its first and second time derivative, was rearranged to find an expression for an eliminated state in terms of the remaining states. The equations of motion for the eliminated states were rearranged to find expressions for the constraint forces in terms of the remaining states, which were substituted into the ODE equations. The equations used to eliminate variables were removed from the DAE, giving a solvable ODE.

Using elimination of variables is inadvisable for most nonlinear DAEs [6] and the GGL formulation [5] is generally preferred. However, the equation considered in this paper is a linear, time-varying DAE. Therefore, the algebraic elimination only had to be performed once and reducing the DAE to an ODE was not computationally expensive. 


\subsection{Reconstruction}

After the ODE had been solved by integration, the solution process was reversed to recover the eliminated states. The eliminated variables were evaluated using the previously evaluated expressions, then the $\psi_{n}(x)$ and $q_{n}(t)$-terms were recombined to find the wire deflections.

\section{Results}

\subsection{Validation Against BS:EN 50318:2002}

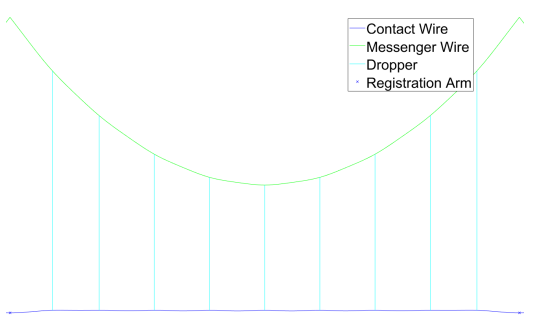

Fig. 3: Diagram of single messenger span for BS:EN 50318:2002

The software was first validated against BS:EN 50318:2002. This involved modelling the interaction between a single pantograph and a simplified catenary system consisting of ten uniform messenger spans, as shown in Figure 3. Transitions and dropper unloading were not required.

The simulation was performed at $250 \mathrm{~km} /$ hour and at $300 \mathrm{~km} /$ hour. The contact-force profile for the central two messenger spans and the time profile of the vertical displacement of the contact wire under each messenger support were recorded and compared to the benchmark, as shown in the tables below.

\begin{tabular}{|c|c|c|}
\hline 250 km/hour & Required & Obtained \\
\hline Mean contact force (N) & $110-120$ & 116.69 \\
Standard deviation of contact force (N) & $26-31$ & 30.88 \\
Mean contact force - 3s.d. (N) & $20-40$ & 24.06 \\
Mean contact force + 3s.d. (N) & $190-210$ & 209.33 \\
Minimum of contact force (N) & $50-75$ & 55.54 \\
Maximum of contact force (N) & $175-210$ & 188.56 \\
Maximum uplift at support (mm) & $48-55$ & 48.04 \\
Percentage loss of contact (\%) & 0 & 0.00 \\
\hline Time taken (s) & - & 5 \\
\hline
\end{tabular}

Table 1: Table of $250 \mathrm{~km} /$ hour results for validation against BS:EN 50318:2002

\begin{tabular}{|c|c|c|}
\hline 300 $\mathbf{~ k m} / \mathbf{h o u r}$ & Required & Obtained \\
\hline Mean contact force (N) & $110-120$ & 116.65 \\
Standard deviation of contact force (N) & $32-40$ & 37.46 \\
Mean contact force - 3s.d. (N) & $-5-20$ & 4.26 \\
Mean contact force + 3s.d. (N) & $210-230$ & 229.04 \\
Minimum of contact force (N) & $30-55$ & 37.17 \\
Maximum of contact force (N) & $190-225$ & 215.47 \\
Maximum uplift at support (mm) & $55-65$ & 55.13 \\
Percentage loss of contact (\%) & 0 & 0.00 \\
\hline Time taken (s) & - & 4 \\
\hline
\end{tabular}

Table 2: Table of $300 \mathrm{~km} /$ hour results for validation against BS:EN 50318:2002 
The results proved to be accurate, with every value falling within the required range. The simulations took $5 \mathrm{~s}$ and $4 \mathrm{~s}$ at $250 \mathrm{~km} /$ hour and $300 \mathrm{~km} /$ hour respectively. This is faster than real time and could be improved even further. These simulations were done in Simulink on a Windows desktop machine with a quadcore processor.

\subsection{Validation Against BS:EN 50318:2018}

The new standard requires the modelling of two pantographs in contact with the same wire, as well as dropper unloading. It does not explicitly require contact-wire transitions to be modelled, but these were found to be necessary in order to accelerate the train before reaching the analysis section, which is ten messenger spans in the middle of the central contact wire. A third contact wire was also included to allow the front pantograph to leave the central wire while the back pantograph was still in the analysis section.

Details of modelling wire transitions are given in [2]. The software must detect contact events, when a pantograph comes into or out of contact with a wire, and update the equations accordingly.

Conservation of momentum was used to resolve velocity discontinuities when coming into contact and these events were successfully incorporated into the model, giving the results in the tables below.

\begin{tabular}{|c|c|c|c|c|}
\hline $\mathbf{2 7 5} \mathbf{~ k m} / \mathbf{h o u r}$ & \multicolumn{2}{|c|}{ Required } & \multicolumn{2}{c|}{ Obtained } \\
\hline & Pan 1 & Pan 2 & Pan 1 & Pan 2 \\
\hline Mean contact force (N) & $137-146$ & $138-147$ & 143.33 & 143.29 \\
\hline s.d. of contact force: $0-20 \mathrm{~Hz}(\mathrm{~N})$ & $31.9-34.8$ & $50.0-54.5$ & 32.59 & 51.70 \\
\hline s.d. of contact force: 0-5Hz (N) & $25.6-28.9$ & $41.2-45.4$ & 26.50 & 44.50 \\
\hline s.d. of contact force: 5-20Hz (N) & $16.2-23.1$ & $25.2-34.7$ & 18.97 & 26.32 \\
\hline Maximum of contact force (N) & $211-244$ & $241-290$ & 235.07 & 244.84 \\
\hline Minimum of contact force (N) & $71-86$ & $14-50$ & 72.52 & 25.32 \\
\hline Contact-point displacement range (mm) & $38-49$ & $53-70$ & 43.18 & 68.97 \\
\hline Maximum uplift at support (mm) & \multicolumn{2}{|c|}{$37-54$} & \multicolumn{3}{|c|}{53.22} \\
\hline Percentage loss of contact (\%) & 0 & 0 & 0.00 & 0.00 \\
\hline Time taken (s) & \multicolumn{3}{|c|}{-} & \multicolumn{3}{|c|}{173} \\
\hline
\end{tabular}

Table 3: Table of $275 \mathrm{~km} / \mathrm{hour}$ results for validation against BS:EN 50318:2018

\begin{tabular}{|c|c|c|c|c|}
\hline $\mathbf{3 2 0} \mathbf{~ k m} / \mathbf{h o u r}$ & \multicolumn{2}{|c|}{ Required } & \multicolumn{2}{c|}{ Obtained } \\
\hline & Pan 1 & Pan 2 & Pan 1 & Pan 2 \\
\hline Mean contact force (N) & $150-173$ & $158.5-172.5$ & 164.93 & 164.55 \\
\hline s.d. of contact force: 0-20Hz (N) & $49-63$ & $30-44$ & 49.96 & 42.19 \\
\hline s.d. of contact force: 0-5Hz (N) & $38-44$ & $14-23$ & 40.62 & 29.45 \\
\hline s.d. of contact force: 5-20Hz (N) & $29-46$ & $25-38$ & 29.09 & 30.22 \\
\hline Maximum of contact force (N) & $280-343$ & $247-317$ & 336.16 & 264.96 \\
\hline Minimum of contact force (N) & $50-82$ & $21-86$ & 77.48 & 62.10 \\
\hline Contact-point displacement range (mm) & $39-51$ & $18-35$ & 50.46 & 31.10 \\
\hline Maximum uplift at support (mm) & \multicolumn{3}{|c|}{$52-64$} & \multicolumn{3}{|c|}{62.01} \\
\hline Percentage loss of contact (\%) & 0 & 0 & 0.00 & 0.00 \\
\hline Time taken (s) & \multicolumn{3}{|c|}{-} & \multicolumn{3}{|c|}{91} \\
\hline
\end{tabular}

Table 4: Table of $320 \mathrm{~km} / \mathrm{hour}$ results for validation against BS:EN 50318:2018

As shown, only one value lay outside the required range. When these benchmark results were created, the dispersal of the indicator values for the back pantograph was relatively large [3], so the larger value shown here is not unrealistic. Furthermore, the benchmarking software dealt with the run-in differently from this software, which could explain the discrepancy in low-frequency dynamics. 


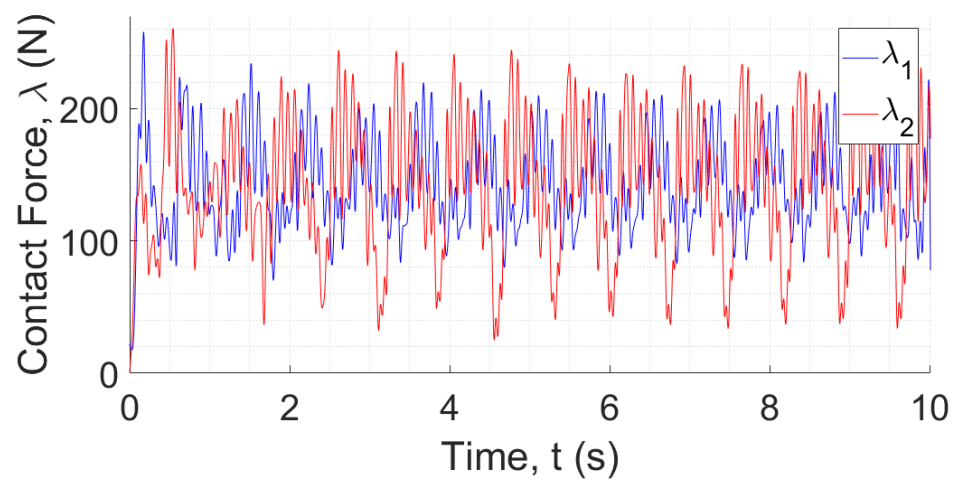

Fig. 4: Plot of contact forces against time for BS:EN 50318:2018 at $275 \mathrm{~km} / \mathrm{hour}$

\section{Conclusions}

The pantograph-catenary interaction was modelled using a Galerkin approach instead of FEM. This yielded high simulation speed while still producing accurate results. Horizontal deflections, dropper unloading and contact-wire transitions are all included in the model. The model allows for multiple pantographs and can take any velocity profile as an input.

The model was validated against BS:EN 50318:2002 and BS:EN 50318:2018. There is further work to be done on this model, but this serves as a proof of concept of the Galerkin approach for modelling the pantograph-catenary interaction.

Current work involves updating the Simulink model to simulate a real train journey. This entails generalising the model to accept catenary systems with different lengths, layouts, material properties, as well as modelling pantograph transitions from one wire to the next. The final model will be validated against line-test data from the Old Dalby track.

\section{References}

1. Jorge Ambrósio, Joao Pombo, Manuel Pereira, Pedro Antunes, and António Mósca. A computational procedure for the dynamic analysis of the catenary-pantograph interaction in high-speed trains. Journal of Theoretical and Applied Mechanics, 50(3):681-699, 2012.

2. Jesús Benet, Angelines Alberto, Enrique Arias, and Tomás Rojo. A mathematical model of the pantograph-catenary dynamic interaction with several contact wires. International Journal of Applied Mathematics, 37(2), 2007.

3. Stefano Bruni, Jorge Ambrosio, Alberto Carnicero, Yong Hyeon Cho, Lars Finner, Mitsuru Ikeda, Sam Young Kwon, Jean-Pierre Massat, Sebastian Stichel, Manuel Tur, et al. The results of the pantograph-catenary interaction benchmark. Vehicle System Dynamics, 53(3):412-435, 2015.

4. Tore Dahlberg. Moving force on an axially loaded beamwith applications to a railway overhead contact wire. Vehicle System Dynamics, 44(8):631-644, 2006.

5. Charles William Gear, Ben Leimkuhler, and Gopal K Gupta. Automatic integration of euler-lagrange equations with constraints. Journal of Computational and Applied Mathematics, 12:77-90, 1985.

6. P. Mantegazza and P. Masarati. Analysis of systems of differential-algebraic equations (dae). Graduate Course on "Multibody System Dynamics", 2012.

7. J Pombo, J Ambrósio, M Pereira, F Rauter, Andrea Collina, and Alan Facchinetti. Influence of the aerodynamic forces on the pantograph-catenary system for high-speed trains. Vehicle System Dynamics, 47(11):1327-1347, 2009.

8. Jong-Hwi Seo, Hiroyuki Sugiyama, and Ahmed A Shabana. Three-dimensional large deformation analysis of the multibody pantograph/catenary systems. Nonlinear Dynamics, 42(2):199-215, 2005. 